\title{
Augmented Analytics and Modern Business Intelligence Adoption to Maximize Business Value
}

\author{
D.O.I - 10.51201/Jusst12685 \\ http://doi.org/10.51201/Jusst12685
}

Mr. Prakash Ukhalkar ${ }^{1 *}$, Dr. Santosh Parakh ${ }^{2}$, Dr. Rajesh Phursule ${ }^{3}$, Mrs. Leena Sanu ${ }^{4}$

${ }^{1}$ Assistant Professor, Department of MCA, Pimpri Chinchwad College of Engineering,

Pune, Maharashtra, India

${ }^{2}$ Associate Professor, Department of MCA, Vidya Pratishthan's Institute of Information and Technology, Baramati, Pune, Maharashtra, India

${ }^{3}$ Associate Professor, Department of IT, Pimpri Chinchwad College of Engineering, Pune, Maharashtra, India

${ }^{4}$ Assistant Professor, Department of Computer Science, Christ College, Pune, Maharashtra, India

\begin{abstract}
The business success is critical for any organization irrespective of its size. Understanding business value and advanced technology capabilities in Business Intelligence (BI) solutions for analysis and decision making is the need of every management, researchers and analysts.

Data is available at every corner, at every platform and it is being produced all the way using digital equipment. These complex data sets for analysis and decision-making can be used on various platforms and are more accessible than ever to find insights and explanations. There is an abundance of datasets and data analysts, but organizations are still unable to leverage its potential to the fullest and this perhaps is the failure of most data analytics initiatives.
\end{abstract}

This paper covers the need for Augmented Analytics adoption in companies to maximize business value with respect to added advanced analytics capabilities in BI Tools for effective, accurate and timely decision making, and business analysis.

Keywords: Business Intelligence, Augmented Analytics, Machine Learning, Natural Language Processing, Citizen Data Scientist.

\section{Introduction}

This Business Intelligence (BI) is a term that refers to the use of data analysis to design strategies and make decisions so that companies have a competitive advantage. Although it can become a product in the digital age, unless it has the ability to deal with changing business needs and make effective decisions based on advanced analysis techniques. The term BI refers to tools designed together with business applications and strategies to collect, integrates, analyze and interpret business information. Its main purpose is to provide better business decision making. Business analysis is a field that uses statistics and qualitative techniques to obtain knowledge from raw data, while the predictive model is a field that uses data analysis techniques to make effective and evidence-based business decisions [1]. 
Traditional BI needs to analyze isolated databases to create basic reports. The analysis is performed by specialized data analysts and access to the reports produced is limited to very few. Regular business users do not have access to data. Hence, they cannot make data-wise decisions. The BI tools are made more accessible to business users with the development of self-service technologies. These tools are equipped with intuitive graphical user interfaces and smart dashboards.

With the help of self-service BI tools, business users can more easily obtain the information they need to make better decisions without relying too much on data analysts and IT professionals. Moreover, compared with traditional BI systems, these tools can handle larger numbers, allowing for more in-depth analysis. Most current BI tools and platforms fall into this category.

Self-service BI tools are more insightful and easier to use than traditional BI, but these tools have some limitations Most of the process of data preparation, structuring, and cleaning for accurate analysis is manual. This manual process of turning raw data into useful insights is usually a lengthy process involving several steps.

In addition to being cumbersome, this manual data analysis process in self-service BI is inefficient and prone to human error. Similarly, the quality of insights provided by Selfservice BI systems is limited to the types of queries that business users make. If users do not query their BI system in the correct way, they may ignore hidden trends and insights, resulting in failure to resolve missed opportunities or alerts in a timely manner. [2]

Therefore, it is important not only to automate data preparation, but also to automate part of the data analysis and insight discovery process. This can be achieved by using Artificial Intelligence (AI), Machine Learning (ML) and other advanced technologies to power BI.

In the following sections, BI and Advanced data analytics approaches have highlighted along with the Augmented Analytics technology, its benefits, essential elements, business adoption and business requirements to focus the need of it in BI tools to automate data analysis, increase business value and for overall organizational growth.

\section{BI and Advanced Data Analytics}

The Gartner [3] in their report have estimated that 85 percent and more projects on Big data are failed. Datasets are increasing in size and becoming more complex and dynamic, and traditional BI systems are not able to manage them. The failure of data analysis is very obvious. They either cannot obtain the data, process, prepare the data, or even cannot understand the data.

Traditionally, the processes involved in preparing, browsing, and manipulating data have been mostly manual. With the rapid growth of data volume, the traditional manual data analysis process has become inefficient due to the complexity brought about by the continuous increase of data volume. In addition to manual operations, the data science process requires specialized data scientists, and these scientists are not many. In fact, there are very few data analysts and scientists available in the global market. Even if the organization manages to obtain the right data experts, they do not have or have little business knowledge, so they need comprehensive training in the analysis required by the business.

Modern BI systems with advanced analytics are capable of analyzing large amounts of data. However, the process before analysis, data cleaning, is still very manual and needs to be performed by data scientists. They have to manually develop metadata and ensure 
data profiling, quality, modeling and manipulation. Consequently the possibility of human error increases even before data analysis.

With Modern BI and analytics platforms, users can easily find the data they are looking for and visually explore data patterns and relationships. But the limitation is that users may not be able to understand hidden data trends and skews that they do not know, and may affect the business. Users continue to focus on data exploration with their own preconceived notions and past experiences or they will have to manually explore all the potential permutations and combinations. This process is obviously time-consuming and users are likely to miss important insights.

Most Modern BI tools offer smart visualizations and interactive dashboards. However, it requires special skills and understanding of what is really important in the data. The [4] has covered four general data analysis types, including descriptive, diagnostic, predictive and prescriptive analysis that organizations can implement together with BI tools and strategies to make better and more effective decisions. These four types are illustrated in the Figure. 1

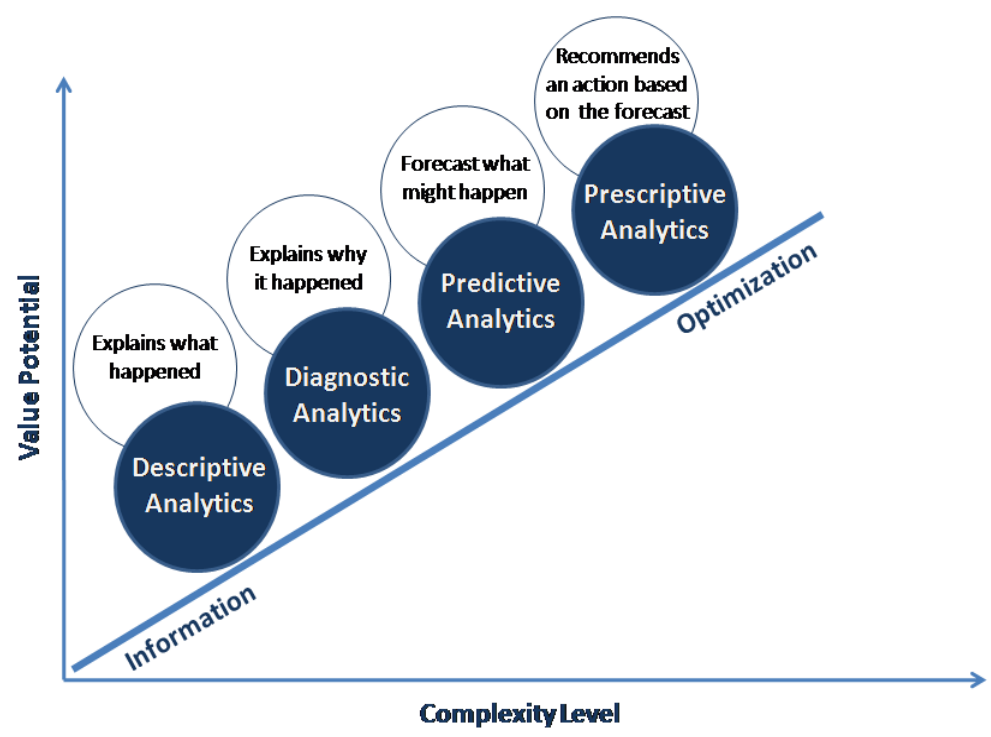

Figure 1. Levels of Data Analytics

Gartner said that Advanced Analytics is a top business priority [5]. In that, Gartner have depicted the four types of Analytics capabilities which are depicted in Fig. 1.

In Descriptive Analytics comprehensive, accurate and past data is processed for business reporting and effective visualization to understand what had happened with the company's business strategies in the past and it explains what was exactly happened with descriptive analytics capability. In case of Diagnostic Analytics, companies can drill down to the root-cause and it can isolates all confounding information to understand what certain things had happened in the past. In order to forecast what will happen in the future, the companies are looking for the Predictive Analytics capabilities to make business strategies that remain fairly consistent over time. In Predictive Analytics, decisions are automated using algorithms and technology. While Prescriptive Analytics, recommends action and business strategies based on competitors testing strategies and outcomes. It an Advanced Analytics technique to make specific recommendations based on the forecast. The prescriptive model utilizes an understanding of previous three models 
descriptive, diagnostic and predictive for what happened, why it happened, and a variety of what will likely to happen analysis to help determine the best course of action to take in the future.

Business leaders want their company to become a data-driven enterprise and recognize the need to adjust Advanced Analytics features to make it a digital business. However, in most cases, the company is simply collecting more data, big data, rather than converting that into actionable insights. According to Gartner [6], 34\% of companies undertake either diagnostic analytics or explore why KPIs are working in a certain way. Although $72 \%$ and $3 \%$ have partially or minimally completed their respective tasks, even lower percentages can fully perform predictive and prescriptive analysis.

Augmented analysis has the potential to improve the company's analytical methods; it will simplify root cause analysis, predictive analysis and prescriptive analysis, and further reduce the burden on data scientists. Even, existing information analysts will evolve into citizen data scientists [7] and they will be able to spend less time on data preparation and descriptive analysis, and more on advanced analysis aided by smarter software and modern BI Tools.

\section{Augmented Analytics: Future of BI}

The Gartner [8] defines Augmented Analytics as a method that uses artificial intelligence (AI), machine learning (ML) and natural language generation (NLG) to automate insights, which will mark the next wave of disruption in the data and analytics market. Organizations should plan to use these supporting technologies to adopt augmented analysis to automate data preparation, integration, insight, and interpretation. By automating many aspects of data analysis, ML, NLG and AI along with BI and associated business strategies, organizations will leverage the potential of it, in making effective decision making and for overall organizational growth.

Gartner have also proposed an Augmented Analytics will be the future of big data and analytics [9] which would further deliver in making improved BI strategies and decision making. Augmented Analytics is an innovative approach to think about data and analytics. It includes AI, ML and Natural Language Processing (NLP), into traditional BI. It is an advanced approach in which AI works in the background to learn and improve the results. It also provides faster access to insights derived from various data. This technology ultimately helps to discover hidden patterns, generate insights, and reduce bias. In addition, companies can collect the most appropriate data model for analysis and understanding of BI insights.

Organizations need to deploy Augmented Analytics capabilities into BI, it will not only democratize the use of the data but also make it simple for business users and experts to make effective decisions based on added advanced analytics functionalities without help from data scientists or business professional. It will also help to go beyond predictions of future business events or situations and obtain unbiased prescriptive analysis to understand what might be done next. That is really the future of BI!

With augmented analytics, business users can:

- Provide unbiased data analysis.

- Easily determine the root cause of the problem.

- Identify unknown business growth opportunities. 
- Democratize company-wide insights from BI to enhance overall business performance.

- Turn actionable insights into opportunities.

- Develop rapidly to build an agile enterprise.

The global market is evolving at an unprecedented pace. Due to changes in markets, regulations, demands, and emergencies such as the COVID-19 pandemic, it is difficult to predict [10]. In this way, the most successful organizations will be those that will adapt innovatively to this change. Augmented Analytics is important for organizations to identify trends at the onset, and even predict or prescribe them before they can happen.

\section{Benefits of Augmented Analytics}

There are various benefits of augmented analytics for an enterprise, including:

- IT support systems can easily focus only on business strategies and special projects.

- ML can effectively analyze big data sources, while Augmented analysis helps to conduct in-depth automated data analysis.

- Simplifies overall data analysis and makes it easier to get to deep insights.

- Users and an enterprise become data-driven.

Augmented analytics will make it easy to handle the complexity and big data scale from in and out of the enterprise to collect, prepare, integrate, analyze and interpret and make it effective for making smarter business decisions well in time.

\section{Essential Elements of Augmented Analytics}

There are elements of an Augmented Analytics tool which will perform augmented data discovery, data science, ML algorithms, and data preparation.

1. NLP: The technical language skills will mainly benefit BI and citizen data scientists. NLP makes it convenient to interact with the system natural language using text and voice commands.

2. NLG: It is another augmented analytics capabilities that empowers BI tools to recite results to better understand the complexities behind the business data.

In order to deal with any deviations, errors in data and improve the effectiveness of BI tools, augmented analytics should be recommended with the following tasks:

- most appropriate visuals for data

- how to enrich and contextualize data for improved analysis and interpretations

- how to cleanse and prepare data for further use

- Insight Generation: BI tools need to provide insights free from bias and suitable for hypotheses testing. Algorithms used in insight generation need to describe the data, identify KPIs, and draw out the variables that influence the performance. It 
also needs to perform outlier analysis to avoid unexpected results than the anticipated one.

- Prediction: In order to predict, these tools should be able to use algorithms to train/test predictive models which are based on common business functions or problems like customer churn, customer behavior, attrition, increase or decrease in sales across various business dimensions.

Integrating Augmented Analytics with AI, ML NLG and NLP into BI are key elements to provide better user experience across business functions and business analytics. The core business processes for data integration, insight generation, and correlations analysis will become efficient with this approach.

It is important from the organizations point of view to adapt augmented analytics platform to extract meaning from complex and ever growing data deluge. The technologies involved in it will make easy to share business findings. To automate several key aspects of business data management including increase in the productivity of data experts, data governance and its accuracy, and reduction in overall costs as well as for many other such business factors Augmented Analytics systems are essential.

\section{Augmented Analytics Adoption}

The Augmented analytics integrates AI, ML and NLP elements into analysis and BI processes to simplify data preparation, discover insights, visualize and share results with business users.

Augmented analytics functionalities will disrupt the analytics market and its adoption will certainly be game changer for business experts particularly for modern BI, advanced analytics and for data science platforms. The key is to automate insights and embed this technology into enterprise applications which will help business transformation. In order to perform analysis, currently, content authors such as data analysts, citizen data scientists, and business experts repeatedly perform data processing, insight generation, and knowledge representation activities to understand hidden insights. The core steps are:

- Preparing the data

- Finding patterns in the data and building models

- Sharing and operationalizing findings from the data

The augmented analytics rapidly perform accurate insights for business users and augments their analysis by using AI-ML-NLP to automate these three analytic processes used in data discovery and visual platforms are illustrated in Figure. 2. Here, business users often iterate between preparing data and finding patterns in data.

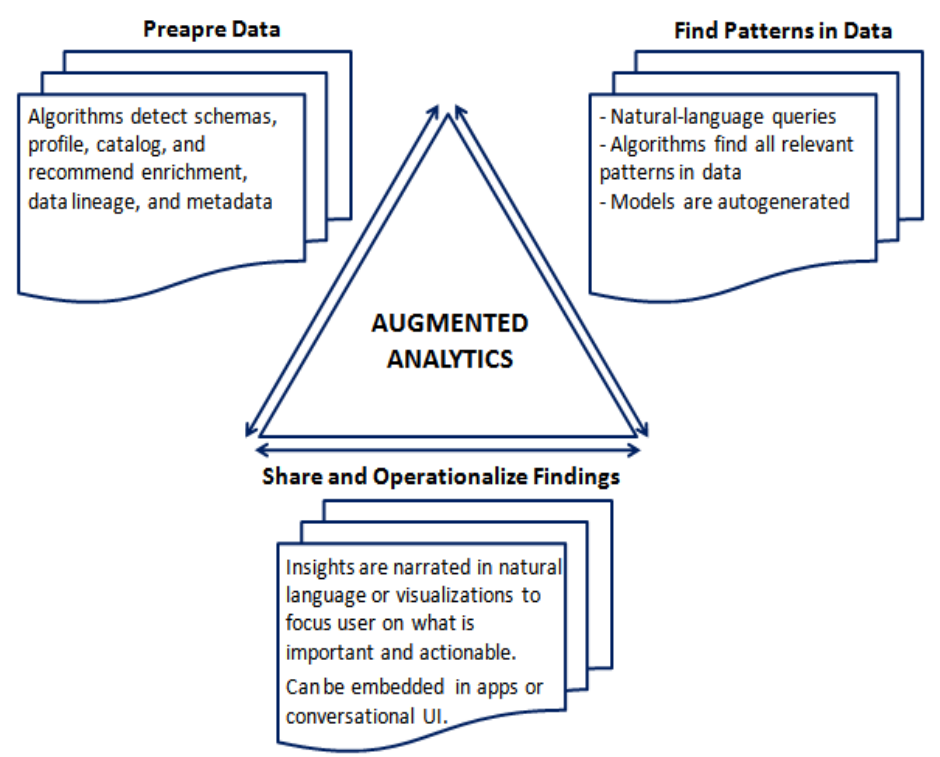

Figure 2. Emerging Augmented Analytics Workflow 


\subsection{Preparing Data}

Data preparation for analysis is the most time-consuming task. Modern BI tools and analytics applications offer basic data preparation functionalities for joining, manipulation and transformation of data.[11] Business users or data scientists have to perform much of the data profiling, maintain quality, its modeling, data manipulation, data enrichment, metadata development and harmonization work. In either way, it creates a bottleneck for users involved in data preparation and also creates risk due to lack of centralization or because of users ability to build analysis content.

One of the components of Augmented analytics is augmented data preparation it performs various tasks to find relationships in data, profiling and recommend the best methods for cleaning, reconciling, enriching, manipulating and modeling data with approaches to capture metadata and lineage for reuse and governance.

\subsection{Finding Patterns in Data}

Modern BI and analysis tools enable business users to use interactive techniques such as data pivoting, sorting, filtering, linking, grouping, and certain custom calculations to view and explore associations and patterns in data. Compared with traditional BI systems, these methods are very effective in providing insights and can promote business development in a highly competitive market. As data becomes complex, large, and multidimensional, the process becomes time-consuming and laborious to explore hypotheses or to infiltrate and combine to ensure complete and accurate results.

On the other hand, in Augmented Analytics, the algorithms used to detect correlations, clusters, outliers and associations are automated and applied to business data. In this case, only statistically meaningful and relevant results can be better explained to users with the help of advanced visualization functions. This can be done by applying multiple algorithms in parallel to reduce the risk of losing valuable insights in the data.

Most of the Augmented Analytics platforms will make the basic analysis model open for testing and verification by business experts. This is one of the important features to build the confidence and confirm the reliability of automated analytics and insights.

AI, ML, and NLP automation in Augmented analytics are entering the field of data science to simplify functional design, model generation, and prediction. This will make professional data scientists more efficient, and the business models they build are less prone to bias.

In view of the lack of professional data scientists in the market and the growing demand for their skills, a new category of citizen data scientists will be expected to achieve higher productivity and require more analytical work.

\subsection{Sharing and Operationalizing Findings From Data}

Modern BI and analytical tools have developed intelligent visualization and interactive dashboards to provide collaboration capabilities to assist in presenting patterns and hidden insights.

With addition of ML, NLG, and NLP, augmented analytics can also automate text or voice narration in data to provide actionable insights to business users. 
Organizations need to adapt Augmented Analytics capabilities to make better business decisions based on automated analytics using AI, ML, NLP algorithms. The following are some necessary suggestions that organizations or business leaders should follow when implementing these technologies on business data.

- Determine the ML features and algorithms for data preparation and integration.

- Plan for augmented analytics in terms of business outcomes, enhance self-service BI and data scientist's skills.

- Define algorithms to automate pattern detection in data which can simplify insight generation and model building.

- Recognize the expert data scientist to interpret and validate models, insights and business applications, they will still need to perform these tasks.

- In order to improve the skills of citizen data scientists, apply the current deployment model through AI-ML-NLP technology, and implement analytical governance among data engineers, analysts and data scientists.

- Execute augmented analytics initiatives along with existing analytics and decision making processes to understand the similarities and differences.

- Encourage business users to work collaboratively and iteratively with expert data scientists both internal, if available, and external.

- First, lists the business problems that are difficult to solve with traditional BI in terms of time, technology, and data processing complexity, and then apply augmented analytics to evaluate its effectiveness.

- When using Augmented analytics tools in learning and business decision-making, assign responsibilities and roles to business analysts and data scientists, and enable them to interact to better understand the overall business process and augmented analytics capabilities.

- Hire people with data analysis skills in the business area and invest in augmented analytics throughout the organization.

\section{Augmented Analytics Risks Factor}

The emergence of visual augmented analytics allows a wide range of non-technical users to access easy-to-use, visually interactive, and more advanced tools to prepare and analyze data, thereby democratizing analysis.

Augmented Analytics will eventually become more accessible and will continue to evolve in terms of technologies and features. Business executives should plan to adopt and understand its impact on BI and analysis. They also need to plan to invest in the development of data analysis skills to enable users to gain insights and develop business strategies to assess the impact on traditional or currently supported BI tools and analysis capabilities.

To date, BI tools need to perform a lot of manual data analysis and business user intervention and interpretation. Looking at volume, complexity, and speed at which big data is generating, this approach of data analysis and insight generation is not suitable or scalable in today's business environment. 
Augmented analytics is the next big thing in the market and it will disrupt the big data and analytics industry. On the other hand, Analytics leaders need to embrace to establish and maintain a competitive advantage, especially in industries that are undergoing digital transformation. Efforts to adopt Augmented analysis may encounter resistance for several reasons:

- Believing in traditional BI and analytical methods, manual data analysis, and assumptions that it can solve business problems and provide feasible insights.

- Less knowledge of Augmented Analytics tools and their functions in terms of effective implementation.

- Employment is unsafe, Augmented analytics may not require manual intervention due to its automated data analysis function.

- Companies rely on traditional decision-making processes and find it difficult to adapt to strategic changes.

- Business executives think that Augmented Analytics is still some time away. Businesses can still consider the existing BI tools with an advanced analytics approach.

These reasons are challenges to Augmented analytics implementation. This paradigm shift needs to be challenged through the existing processes and strategies of decisionmaking and business analysis, and needs to prove the similarities and differences between traditional BI and Augmented Analytics. Organizations need to create an environment to support and adapt the Augmented Analytics in an effective way that it is has potential and can create business value. This will further help in identify business issues that cannot be seen using traditional BI, and will also help deliver relevant results within an appropriate time frame. This will lay the foundation for futuristic automated data analysis and decision-making methods in BI.

\section{Augmented Analytics Tools}

There are many BI systems with overlapping functions in the market, but they are different in terms of providing services to different users. Analytics companies have developed products that can provide advanced analysis and automated data discovery, pattern recognition, smart visualizations and interpretations. For example, Watson Analytics from IBM, Lumira from SAP, Power BI from Microsoft and Visual Analytics from SAS offers advanced analytics capabilities for Augmented Analytics. Some companies have begun to invest in NLG, including Narrative Science, Yseop and Automated Insights. Tableau, Qlik and many other such tools are not behind to invest in Augmented Analytics.

There are major players in the market, such as Datawatch, Paxata, UniFi, Trifacta, Alation, Informatica and Oracle, which provide automated data preparation functions. However, these functions are still in its infancy and adoption is in the early stages. Most of these tools can run advanced analysis algorithms and allow expert data scientists to evaluate the model and provide it for further enhancements, making it a high-level descriptive, predictive and prescriptive model.

A number of AI, ML and data science platforms, Visual Statistics and SAS Visual Data Mining and Machine Learning, RapidMiner and KNIME need little skills to build advanced analytics models. These tools include advanced analytic techniques such as semantic analysis, graph analysis, ML, NLG, NLP, pattern matching, sentiment analysis, 
network and cluster analysis, forecasting, smart visualization, advanced statistics, neural networks and other complex features along with the programming languages or packages such as open source R, Python and MATLAB support.

Compared with traditional analysis methods, the Augmented analysis platform is unique because it can automatically execute the analysis process at a faster speed than humans and avoid decision bias [12]. Although Augmented analytics development is in progress and it is still at an early stage compared with disciplines such as BI tools.

Major Software providers offering Augmented analytics services in the market [13] include IBM, SAP, Salesforce, Qlik, Microsoft, Oracle, SAS, TIBCO, dotData, Aible, AnswerRocket, Big Squid, DataRobot, Outlier, Prevedere, Tellius, Evolved Analytics DataStories, H2O.ai, and Sisense.[14]

Augmented analytics is augmented intelligence, it supports AI, ML, NLP, NLG and advanced analytics algorithms including descriptive, diagnostic, predictive and prescriptive. Gartner described it as a technology for humans and machine partnerships [15]. Products such as Siri from Apple and Alexa from Amazon, are great examples of augmented intelligence having AI and NLG technologies embedded in it. [16]

\section{Conclusion}

The Augmented Analytics provides effortless and automated data collection, preparation, integration, analysis, visualization and interpretation to the business system. The BI vendors have designed tools to adopt Augmented Analytics and Advanced Analytics capabilities to make industry future ready though the development of these tools are in the early stages. The business industry can leverage its full potential unless it is implemented and adopted by moving away with traditional BI and decision making process. The adoption of Augmented analytics will bring business opportunities and consensus among business users, little knowledge and training in the use of these tools can further realized it. In this paper we have documented the need of Augmented Analytics adoption in businesses to maximize business value, it is high time for Data Analytics leaders and Business Industry to shift focus on to this automated tools and techniques for Business Data Analysis. We have also highlighted how Augmented Analytics can be seen as the Future of BI and how organizations can lead in terms of faster, more accurate and automated data analysis processes using AI, ML, NLP, NLG, and using other such important technologies, and tools with Augmented Analytics capabilities. There is a way ahead for full implementation of Augmented Analytics in business industry but the future is not too far to adopt as it has immense value potentials in industry.

\section{REFERENCES}

[1] Jianlong Zhou, Syed Z. Arshad, Xiuying Wang, Zhidong Li, Dagan Feng, Fang Chen, "End-User Development for Interactive Data Analytics: Uncertainty, Correlation and User Confidence", IEEE Transactions on Effective Computing, Vol. 9, No. 3, pp. 383395, July-September 2018.

[2] Romil Shah, Going beyond Business Intelligence with Augmented Analytics. [Online]. Available: $\quad$ https://phrazor.ai/blog/augmented-analytics-futuristic-businessintelligence. [Accessed 25 - January - 2021]. 
[3] Daniel Newman, 5 Fixes For Your Failing Big Data Initiatives. [Online]. Available: https://www.forbes.com/sites/danielnewman/2018/06/28/5-fixes-for-your-failing-bigdatainitiatives/\#a5f245a5a0bf. [Accessed 27 - January - 2020].

[4] Prakash K. Ukhalkar, Dr. Rajesh N. Phursule, Dr Devendra P Gadekar, Dr Nilesh P Sable, "Business Intelligence and Analytics: Challenges and Opportunities", IJAST, vol. 29, no. 12s, pp. 2669-2676, June 2020.

[5] Gartner, Analysts to Explore Analytics Capabilities at the Gartner Business Intelligence \& Analytics Summit, 2014. [Online]. Available: https://www.gartner.com/en/newsroom/press-releases/2014-10-21-gartner-saysadvanced-analytics-is-a-top-business-priority [ Accessed 05 - February - 2021]

[6] Rita Sallam, Cindi Howson, Carlie Idoine, Augmented Analytics Is the Future of Data and Analytics, Garter $2017 . \quad$ [Online]. Available: https://www.gartner.com/en/documents/3773164/augmented-analytics-is-the-futureof-data-and-analytics. [Accessed 08 - February -2021].

[7] Carlie Idoine, Citizen Data Scientists and Why They Matter, 2018. [Online]. Available: https://blogs.gartner.com/carlie-idoine/2018/05/13/citizen-data-scientistsand-why-they-matter/. [Accessed 13-February-2020].

[8] Gartner IT Glossary, Augmented Analytics, [Online]. Available: https://www.gartner.com/en/information-technology/glossary/augmented-analytics. [Accessed 17-February-2020].

[9] Gartner Data and Analytics Summit, Augmented Analytics Is the Future of Analytics 2020. [Online]. Available: https://www.gartner.com/en/conferences/apac/dataanalytics-india/gartner-insights/gc-augmented-analytics [Accessed: 25-February20201.

[10] Rajesh N. Phursule, Parikshit N. Mahalle, Prakash K. Ukhalkar, Satish R. Todmal, "Machine Learning-Based IoT-Enabled Perspective Model for Prediction of COVID-19 Test in Early Stage”, IJAST, vol. 29, no. 12s, pp. 2599-2604, June 2020.

[11] Prakash Ukhalkar, The transformative potential benefits of big data in government and public sector domains, International Journal of Advanced Science and Research, Vol 3, Special Issue 1, 2018, Pg. 30-33

[12] Pete Reilly, Augmented Analytics 101: A Starter's Guide, 2019 [Online] Available: https://www.selecthub.com/business-analytics/augmented-analytics-guide. [ Accessed: 27-February-2021].

[13] Austin Kronz, Gartner Research, Market Guide for Augmented Analytics, 2019 [Online]. Available: https://www.gartner.com/en/documents/3970874/market-guidefor-augmented-analytics-tools. [Accessed: 01-March-2021].

[14] Prakash Ukhalkar, Manisha Bhosale, "The role of Big data in enhancing business value through Business Intelligence and Big Data Analytics", Our Heritage, Vol-68, Special Issue-27, pp. 83-91, February- 2020.

[15] Gartner IT Glossary: Augmented Intelligence; 2020. [Online]. Available: https://www.gartner.com/en/informationtechnology/glossary/augmented-intelligence. [Accessed: 02-March-2021].

[16] Jost Hoppermann, Benjamin Ensor, Srividya Sridharan, Brandon Purcell, Luis Deya, Andrew Reese. Look For Banking Applications With Embedded Artificial Intelligence: Off-The-Shelf Capabilities Offer A Fast Path Toward AI, 2018. [Online]. Available: https://www.forrester.com/report/Look + For + Banking + Applications + With + Embedde $d+$ Artificial+Intelligence/-/E-RES141762\#. [Accessed: 05-March-2021]. 